\title{
BMJ Open Effects of photobiomodulation therapy combined to static magnetic field in strength training and detraining in humans: protocol for a randomised placebo-controlled trial
}

\author{
Paulo Roberto Vicente de Paiva, ${ }^{1}$ Heliodora Leão Casalechi, ${ }^{1}$ \\ Shaiane Silva Tomazoni, ${ }^{2}$ Caroline dos Santos Monteiro Machado, ${ }^{1}$ \\ Adriane Aver Vanin, ${ }^{2}$ Bruno Manfredini Baroni, ${ }^{3}$ \\ Paulo de Tarso Camillo de Carvalho, ${ }^{1}$ Ernesto Cesar Pinto Leal-Junior (iD ${ }^{1}$
}

To cite: de Paiva PRV, Casalechi HL, Tomazoni SS, et al. Effects of photobiomodulation therapy combined to static magnetic field in strength training and detraining in humans: protocol for a randomised placebocontrolled trial. BMJ Open 2019;9:e030194. doi:10.1136/ bmjopen-2019-030194

- Prepublication history for this paper is available online. To view these files, please visit the journal online (http://dx.doi. org/10.1136/bmjopen-2019030194).

Received 03 March 2019 Revised 25 September 2019 Accepted 04 0ctober 2019

D Check for updates

(C) Author(s) (or their employer(s)) 2019. Re-use permitted under CC BY-NC. No commercial re-use. See rights and permissions. Published by BMJ.

For numbered affiliations see end of article.

\section{Correspondence to} Dr Ernesto Cesar Pinto LealJunior;

ernesto.leal.junior@gmail.com

\section{ABSTRACT}

Introduction In recent years, it has been demonstrated that photobiomodulation therapy (PBMT) using lowlevel laser therapy and/or light-emitting diode therapy combined to static magnetic field (sMF) has ergogenic effects, improving muscular performance and accelerating postexercise recovery. However, many aspects related to these effects and its clinical applicability remain unknown. Therefore, the aim of this project is to evaluate the ergogenic effects of PBMT/sMF in detraining after a strength-training protocol.

Methods and analysis The study will be a randomised, triple-blind, placebo-controlled clinical trial. Healthy male volunteers will be randomly distributed into four experimental groups: PBMT/sMF before training sessions + PBMT/sMF during detraining, PBMT/sMF before training sessions + placebo during detraining, placebo before training sessions + PBMT/sMF during detraining and placebo before training sessions + placebo during detraining. Strength-training sessions will be carried out over 12 weeks, and the detraining period will occur during the 4 weeks after. The muscular strength and the structural properties of quadriceps will be analysed. Ethics and dissemination This study was approved by the Research Ethics Committee of Nove de Julho University. The results from this study will be disseminated through scientific publications in international peerreviewed journals and presented at national and international scientific meetings. Trial registration number NCT03858179.

\section{INTRODUCTION}

Regular physical activity is recommended for the improvement of general health, performance and rehabilitation of chronic diseases. ${ }^{1}{ }^{2}$ Strength training promotes increased strength, muscle mass and functional capacity. ${ }^{3-5}$ However, it is common practitioners interrupt their activities due aspects such as illness or injuries, leading to
Strengths and limitations of this study

- This study has high methodological quality since it is a randomised, controlled and prospectively registered clinical trial.

- Another strength is that it has a triple-blind study design, which means that outcome assessors, therapists and patients will be blinded to interventions over the course of the study.

- The sample size was calculated based on a pilot study to provide the appropriate statistical power to detect precise differences in the primary outcome of the study.

The doses, sites of application and the most effective timepoint for application of photobiomodulation therapy/static magnetic field were previously optimised and published in international peer-reviewed journals.

- A limitation of this study is that only 4 weeks of detraining after 12 weeks of strength training will be analysed.

loss of gains obtained during the training period. ${ }^{6} 7$ These breaks may represent a problem in clinical practice, due the wide use of strength training for different aims as mentioned before. To the best of our knowledge, currently there is no clinical evidence or consensus on any particular resource or method to decrease the effects of detraining (or deconditioning).

Photobiomodulation therapy (PBMT), also known as phototherapy, is a nonthermal process where light interacts with chromophores leading to photophysical and photochemical reactions in different tissues. PBMT is a light therapy that uses non-ionising light sources, such as lasers, light-emitting diodes 
(LEDs) and broadband light, from the visible to the infrared spectrum. ${ }^{8}$ The first report of PBMT-promoted ergogenic effects published 13 years ago ${ }^{9}$ showed that PBMT significantly increased muscle performance when applied before a tetanic contraction protocol to the anterior tibial muscle of rats. In addition to promoting ergogenic effects, PBMT also had a protective effect on the skeletal muscles of the animals, as creatine kinase activity was significantly lower in the irradiated animals than in the non-irradiated animals. Subsequently, a series of clinical trials involving athletes from different sports and healthy individuals conducted by our research group and other groups showed that, when applied before an exercise session, both PBMT and PBMT combined to static magnetic field (PBMT/sMF) can increase the number of repetitions performed, ${ }^{10-12}$ lengthen the time to exhaustion, ${ }^{1013-15}$ increase peak torque ${ }^{1216-19}$ and even improve performance of athletes in field tests, ${ }^{20}$ and in simulated ${ }^{21}$ and real matches. ${ }^{22}$ In many of these clinical trials, when PBMT or PBMT/sMF were applied before exercises, it also had protective effects on the skeletal muscle tissue, allowing a faster recovery. More recently, studies have also shown that PBMT/sMF can potentiate gains in different training protocols, such as strength training ${ }^{23}{ }^{24}$ and aerobic training. ${ }^{25}$

Despite the surprising, remarkable and rapid progress of knowledge in this field, which culminated in the publication of three systematic reviews with meta-analyses ${ }^{26-28}$ highlighting not only the ergogenic effects of PBMT and PBMT/sMF but also its protective effects, ${ }^{28}$ there are some key aspects that remain unknown and must be investigated. Among them, we highlight the potentially beneficial effects of PBMT/sMF during the detraining period after strength-training protocols.

An interesting study conducted by Nakano et $a l^{29}$ showed that PBMT minimised disuse atrophy in rats when used during the active recovery phase of the study from the passive disuse. The authors attributed these beneficial results from PBMT to satellite cell proliferation and angiogenesis. The authors also observed marked satellite cell activation in irradiated animals, which may be related to the increased myoblast proliferation also observed in this study. These findings of Nakano et $a l^{29}$ may indicate that PBMT could in some way be helpful in delaying muscular strength loss during the detraining period after a strength-training programme, as the muscles still would be in activity (ie, would not be in complete inactivity nor immobilised). It is known that in both disuse atrophy ${ }^{30}$ and detraining, ${ }^{31}$ there is a decrease in the activation of satellite cells and a decrease in the cross-sectional muscle area resulting in a consequent decrease in muscle volume and strength. Considering that these events occur in a similar manner both in disuse atrophy and in detraining, and that PBMT had already demonstrated beneficial effects on these aspects when used in an experimental model of disuse atrophy, ${ }^{29}$ it seems reasonable to suspect that PBMT and PBMT/sMF may also be able to attenuate these events when triggered by detraining, since in both situations these events are triggered by decreased muscle activity.

Therefore, the effects of PBMT and PBMT/sMF strength detraining remain unknown and are a scientific challenge that will represent a key advance in the knowledge of this field when it is overcome. With this aspect in mind, we aim to assess the ability of PBMT/sMF for maintaining strength during detraining period after completing a strength-training protocol.

\section{MATERIALS AND METHODS}

A randomised, triple-blind (volunteers, therapists and assessors), placebo-controlled clinical trial will be performed at Laboratory of Phototherapy and Innovative Technologies in Health (LaPIT). The study will follow the ethical guidelines and was approved by the Research Ethics Committee of Nove de Julho University (protocol number 1781602), furthermore this protocol was prospectively registered at ClinicalTrials.org. All volunteers will sign an informed consent at the time of enrolment in this study. This study started on 20 March 2019 and is on schedule to be completed by fifth February 2020.

\section{Subjects and sample size}

As far as we know there are no published studies assessing the effects of PBMT/sMF during the detraining period after a strength-training programme. Therefore, the number of participants per group in the present study was calculated based on a pilot study that was recently conducted by our research group that had five volunteers per group. A $\beta$ value of $20 \%$ and an $\alpha$ of $5 \%$ were used to calculate the sample size.

The pilot study showed that applying PBMT/sMF during the detraining period resulted in a peak torque (the primary outcome of this study) of $257.25 \mathrm{Nm}$ (SD: 33.73) during the maximum voluntary contraction (MVC) test, whereas applying the placebo during the detraining period resulted in a peak torque of $222.05 \mathrm{Nm}$ (SD: 29.87). We used the Researcher's Toolkit to calculate the sample size (https://www.dssresearch.com/KnowledgeCenter/toolkitcalculators/samplesizecalculators. aspx).

Based on the aforementioned parameters used to calculate the sample, we found an $n$ of 10 volunteers per group, for a total of 40 volunteers, was required. Therefore, predicting a $20 \%$ sample loss, 48 healthy, male volunteers aged from 18 to 35 years will be recruited for the study (12 volunteers per group). The choice to recruit only male volunteers is to allow direct comparison with a previous study carried out by our research group. ${ }^{24}$ As previously demonstrated by Joensen et $a l^{22}$ some PBMT devices can significantly increase skin temperature, promote discomfort and even lead to interruption of treatment/irradiation in patients with darker skin. However, since the PBMT/sMF device used in the study does not cause any significant increase in skin temperature nor harmful thermal effects in light, medium or dark 
human skin ${ }^{33}$ volunteers of different skin colours will be recruited. Volunteers will be informed about all study procedures and asked to sign the informed consent form prior to their enrolment in the study.

\section{Patient and public involvement}

Patients and/or the public were not involved in the design, recruitment or conduction of this study. At the end of the study, the main results will be disseminated to participants by email.

\section{Inclusion and exclusion criteria}

Healthy men aged from 18 to 35 years, without training or involvement in a regular exercise programme (ie, exercise more than once per week), with no history of musculoskeletal injury in the trunk, hip, knee or ankle regions in the 2 months before the study, and who do not regularly use pharmacological agents and/or nutritional supplements, will be included in the study. Volunteers who show any musculoskeletal injury that limits or precludes performing the exercises in the strengthtraining protocol in the 2 months before the study or who become injured during the study (self-reported by the volunteer or diagnosed by a medical doctor), who regularly use any type of nutritional supplement or pharmacological agent or who show signs and symptoms of any neurological, metabolic, inflammatory, pulmonary, oncological or cardiovascular disease that may limit the execution of high-intensity exercises will be excluded from the study.

\section{Randomisation and blinding}

Randomisation labels will be created through the random. org website, and a series of sealed, opaque and numbered envelopes will be used to ensure confidentiality and will determine to which experimental group each volunteer will be allocated (4 different experimental groups-12 volunteers in each group). Volunteers will be allocated as described below:

1. PBMT/sMF before the strength-training sessions (12 weeks, 2 times a week) and PBMT/sMF during the detraining period ( 4 weeks, 2 times a week).

2. PBMT/sMF before the strength-training sessions (12 weeks, 2 times a week) and placebo during the detraining period (4 weeks, 2 times a week).

3. Placebo before the strength-training sessions (12 weeks, 2 times a week) and PBMT/sMF during the detraining period ( 4 weeks, 2 times a week).

4. Placebo before the strength-training sessions (12 weeks, 2 times a week) and placebo during the detraining period (4 weeks, 2 times a week).

A researcher will programme the device (placebo or $\mathrm{PBMT} / \mathrm{sMF}$ ) and will be instructed not to inform the volunteers or other researchers as to the type of treatment (PBMT/sMF or placebo). Therefore, the researcher responsible for the treatment will be blinded to the type of treatment being administered to the volunteers. The sounds and signals emitted from the device as well as the information displayed on the screen will be identical, regardless of the type of treatment (placebo or PBMT/ sMF).

\section{Experimental groups}

The individuals randomly allocated to the different groups will be subjected to 12 consecutive weeks of dynamic strength training involving leg-press and knee extension exercises in leg-press and leg-extension machines, respectively, 2 times a week.

As mentioned before, each volunteer will undergo PBMT/sMF or placebo before performing the exercises depending on the group to which he is allocated.

After the 12-week training period, the volunteers will undergo PBMT/sMF or placebo depending on the group to which they are allocated to for 4 weeks (2 times a week) without training. During the detraining period, volunteers will be instructed and monitored to not perform any regular training protocols nor recreational physical activity more than once per week. A flowchart summarising the procedures of this study is presented in figure 1 .

\section{Outcomes}

The protocol includes five planned evaluation visits at baseline, 4, 8, 12 and 16 weeks. All evaluations will be performed at least 24 hours before (baseline) or after 4 , 8, 12 and 16 weeks of any kind of intervention (PBMT/ sMF, placebo or strength training). Evaluations will be performed in the morning and will include image acquisition by echography of the quadriceps femoris muscle and the MVC test. The one-repetition maximum (1RM) test will be performed on the same day, in the afternoon. Volunteers will always be evaluated and re-evaluated at the same time of day to preclude circadian effects on the findings. Volunteers will be instructed to maintain their usual physical and nutritional activities, to avoid drinking alcohol and to sleep well.

All evaluations will be performed by the same researcher who will be blinded to the allocation of the individuals to the different experimental groups. All evaluations will be performed on days other than the strength-training days.

\section{MVC test}

The volunteers will be seated in the isokinetic dynamometer chair (Biodex System 4, Biodex Medical Systems, Shirley, New York, USA) at an angle of $100^{\circ}$ between the trunk and hip, with the tested lower limb positioned at $60^{\circ}$ knee flexion (considering $0^{\circ}$ total knee extension) and fixed to the dynamometer seat using a belt. The other lower limb will also be positioned at $100^{\circ}$ hip flexion and fixed to the seat with a belt. The volunteers will be attached to the dynamometer seat using two belts crossing their trunk. During the tests, the volunteers will be instructed to place their arms across their chest, and the axis of the dynamometer will be positioned in-line with the knee joint line. 


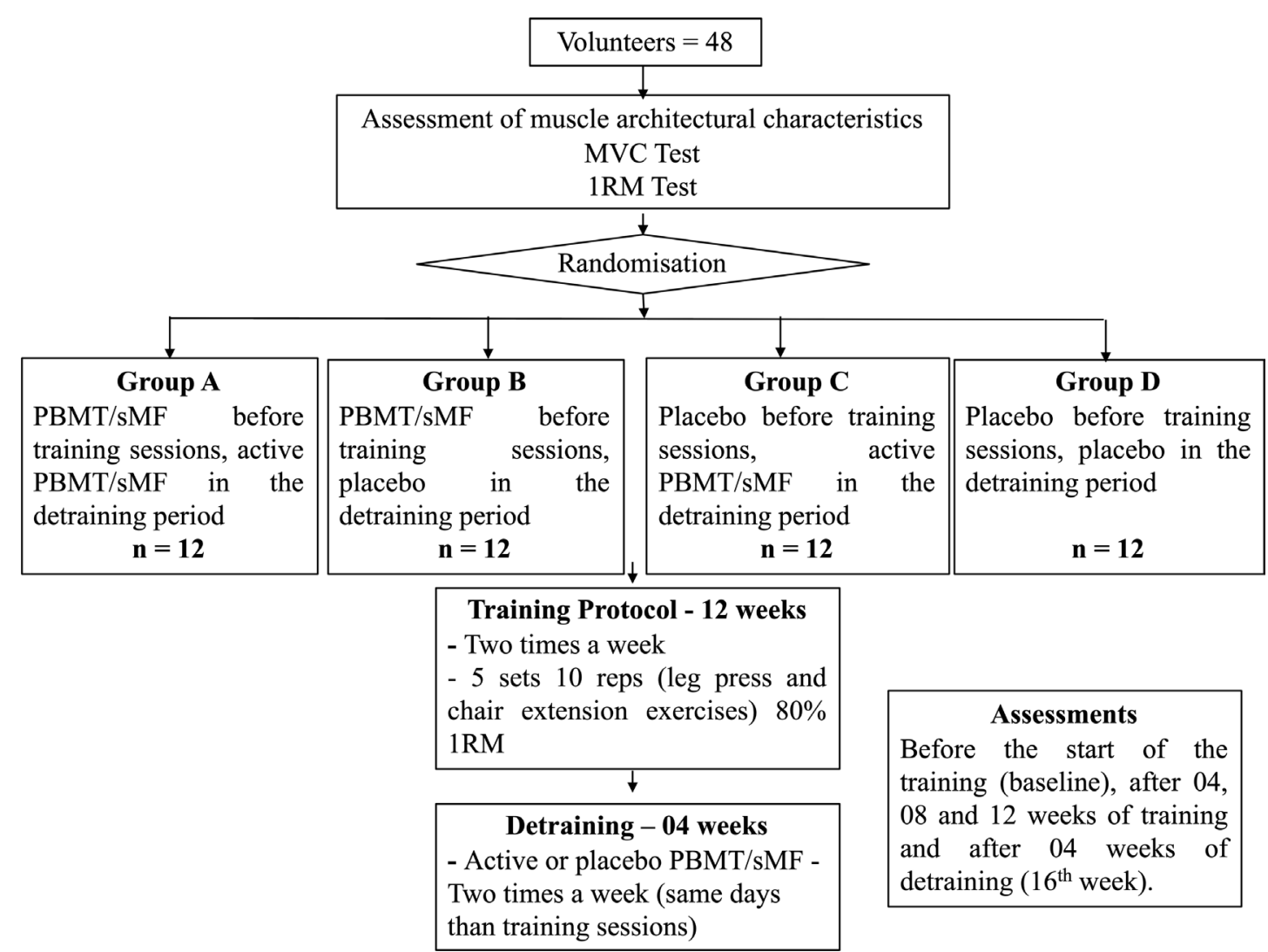

Figure 1 Flowchart summarising the procedures. 1RM, one-repetition maximum; MVC,maximum voluntary contraction; PBMT, photobiomodulation therapy; sMF, staticmagnetic field.

The MVC test will consist of 3, $5 \mathrm{~s}$ isometric contractions of the knee extensors of the lower limb. The highest value of torque assessed in the three contractions (isometric peak torque) will be used for statistical analysis. This parameter will be chosen because this variable reflects the maximal ability of the muscle to generate force. ${ }^{34}$ The volunteers will be verbally encouraged when performing the MVC test and instructed on how to perform the test beforehand.

The MVC test will be performed before starting the study (baseline), at 4,8 and 12 weeks after starting the training period, and at 4 weeks after completing the training (detraining period), in both lower limbs (unilaterally).

\section{RM test}

Initially, the volunteers will be instructed to briefly warm up on a stationary bicycle (Inbramed, Brasil), at $100 \mathrm{rpm}$ and without load, for $5 \mathrm{~min}$.

The proposed range of motion will be from $0^{\circ}$ (full knee extension) to $90^{\circ}$, for both exercises proposed. The anatomical references for the identification of the angle of movement are the greater trochanter of the femur, lateral epicondyle of the femur and lateral malleolus.

Before starting the test, the volunteers will be familiarised performing one set of five repetitions with an estimated load of $<60 \% 1 \mathrm{RM}$. The subjective load will be identified using the OMNI perceived exertion scale $(0=$ extremely easy and $10=$ extremely difficult $) .{ }^{35}$
The 1RM will be determined by a gradual increase in load until the subject is unable to perform the exercise with a full range of motion ${ }^{36}$ and by using the OMNI scale. ${ }^{35}$ The load will be chosen within five attempts, with a 5-min interval between attempts, to avoid metabolic disorders and interferences in test quality. Volunteers will be verbally encouraged to achieve maximum effort, and the test will be performed using both the leg-extension and the leg-press machines (unilaterally).

The 1RM test will be performed before starting the study (baseline), at 4, 8 and 12 weeks after starting the training period, and at 4 weeks after completing the training (detraining period), in both lower limbs. The training load will be reset at 4 and 8 weeks after starting the training period based on 1RM test as well.

\section{Evaluation of structural properties of the quadriceps}

Structural adaptations of the quadriceps muscles to training will be evaluated by ultrasound imaging. An ultrasound device and a $60 \mathrm{~mm}, 7.5 \mathrm{MHz}$ linear array probe will be used to acquire the ultrasound images for the analysis of the muscle architecture of the volunteers. A single researcher who is trained and experienced in the method will be responsible for recording all images, which will be acquired with the muscles at rest and with an adequate amount of water-soluble transmitting gel on the probe. This gel enhances the acoustic contact between the probe and the skin and prevents direct contact and 
possible tissue deformation resulting from the pressure exerted by the probe. ${ }^{37}$

In knee extensor muscles, images will be acquired using the ultrasound probe positioned in the longitudinal direction of the muscle fibres of the rectus femoris and vastus lateralis. The images will be acquired with the individual lying in the supine position on the examination table, with knees fully extended and hips in the neutral position. The probe will be positioned over the rectus femoris and vastus lateralis muscles at the respective midpoints between the greater trochanter and the lateral condyle of the femur. ${ }^{38}$ Maps will be drawn on overhead projector transparencies to ensure that all images are collected at the same points during the five planned evaluations (baseline, 4, 8, 12 and 16 weeks). These images will be used to assess the following muscle architecture parameters: (1) muscle thickness, which is measured along a perpendicular line between the superficial and the deep aponeurosis; (2) fascicle length, which represents the length of the muscle fibre and is a parameter that expresses the number of sarcomeres aligned in series; and (3) fibre pennation angle, calculated by measuring the angle formed between the deep aponeurosis and the fascicle, which indicates the size of the muscular fibres (ie, the number of sarcomeres aligned in parallel).

Furthermore, images of the rectus femoris and vastus medialis muscles will be acquired with the probe positioned transversely to the muscle fibres. These images will be collected at the midpoint between the greater trochanter and the intercondylar fossa of the femur between the femur and the tibia to calculate the anatomical cross-sectional area of the rectus femoris muscle and to estimate the quadriceps muscle volume using the equation proposed by Miyatami $e t a \vec{l}^{39}$ :

$\mathrm{V}=113.7 \mathrm{X}+11.6 \mathrm{Y}-443.7$

Wherein:

$\mathrm{V}=$ volume

$\mathrm{X}=$ quadriceps muscle thickness (rectus femoris + vastus medialis)

Y=segment length.

The method described above was recently used in studies assessing the structural properties of knee extensors. $^{4041}$

\section{Interventions}

\section{Strength training}

After 2 days of baseline evaluation, the volunteers will start the strength training, which will be based on a previous study performed by our research group. ${ }^{22}$ The training will be performed unilaterally (with both right and left legs) with a load of $80 \%$ 1RM, 2 times a week on non-consecutive days (72 hours apart) and will consist of 5 sets of 10 repetitions for 12 weeks (3 months), totalling 24 leg-press and leg-extension workouts. The rest interval between sets will be $2 \mathrm{~min}$ and, if the participant fails to complete a set, the volunteer will be instructed to continue until concentric muscle contraction fails (concentric failure). Room temperature will be kept between $22^{\circ} \mathrm{C}$ and $24^{\circ} \mathrm{C}$, and the load will be adjusted every eight workouts ( 4 weeks).

\section{PBMT combined to sMF and placebo}

$\mathrm{PBMT} / \mathrm{sMF}$ or placebo will be applied before each workout and during the detraining period, depending on the group to which the volunteers are allocated. PBMT/ sMF will be applied bilaterally using the direct contact method with light pressure on the skin to six sites of the anterior thigh (two medial, two lateral and two central).

A 12-diode cluster, with four $905 \mathrm{~nm}$ laser diodes (12.5 W peak power for each diode), four $875 \mathrm{~nm}$ LEDs (17.5 mW mean power for each diode), four $640 \mathrm{~nm}$ LEDs ( $15 \mathrm{~mW}$ mean power for each diode) and $35 \mathrm{mT}$ sMF, manufactured by Multi Radiance Medical (Solon, Ohio, USA), will be used to apply the PBMT/sMF. Considering the large irradiation area used in the present project, the use of diode clusters will be essential for the application of therapy.

The PBMT/sMF dose used for applications during the training and/or detraining periods will be 30 Joules $(\mathrm{J})$ per site ( $180 \mathrm{~J}$ per thigh). The dose was previously established and tested in a study published by Antonialli et $a{ }^{18}{ }^{18}$ in which the same device proposed in the present study was used. The dose, sites and irradiation protocol (PBMT/ sMF before exercise) were tested in a study by Vanin $e t a l^{24}$ and found to significantly increase the muscle strength of the volunteers. For the placebo treatments, the $905 \mathrm{~nm}$ laser diodes, the $875 \mathrm{~nm}$ LEDs and the magnetic field will be deactivated (turned off), and the power of the $640 \mathrm{~nm}$ LEDs will be decreased to $1 \mathrm{~mW}$ (mean power for each diode) in order to keep the visual aspect of red light, but not to deliver an effective therapeutic or considerable dose according the current available evidence. ${ }^{8}$ It is important to mention that this placebo method has been employed in several studies performed previously using the same PBMT/sMF device 1820222425

The sounds and signals emitted from the device as well as the information displayed on the screen will be identical, regardless of the type of treatment (placebo or PBMT/ sMF). PBMT/sMF will be applied by a single researcher who will be blinded to the randomisation results and the volunteer group allocation. Detailed PBMT/sMF and placebo parameters are outlined in table 1.

\section{Statistical analysis}

The primary outcome is MVC. The secondary outcomes are 1RM test and structural properties of the quadriceps. The intention-to-treat analysis will be followed a priori. All the data will be analysed by a blinded researcher not involved in data collection. The findings will be tested for their normality using the Shapiro-Wilk test. Parametric data will be expressed as mean and SD and non-parametric data as median and respective upper and lower limits. Parametric data will be analysed by two-way repeated measures analysis of variance (time vs experimental group) with post hoc Bonferroni correction. Non-parametric data will be analysed using the Friedman 


\begin{tabular}{lll}
\hline Table 1 & Parameters for PBMT/sMF and placebo \\
\hline & PBMT/sMF & Placebo \\
\hline No of lasers & $\begin{array}{l}\text { 4 Super-pulsed } \\
\text { (infrared) }\end{array}$ & $\begin{array}{l}\text { 4 Super-pulsed } \\
\text { (infrared) }\end{array}$ \\
Wavelength $(\mathrm{nm})$ & $905( \pm 1)$ & $905( \pm 1)$ \\
Frequency $(\mathrm{Hz})$ & 250 & 0 \\
$\begin{array}{l}\text { Peak power }(\mathrm{W})- \\
\text { each }\end{array}$ & 12.5 & 0
\end{tabular}

$\begin{array}{lll}\begin{array}{l}\text { Average mean optical } \\ \text { output }(\mathrm{mW}) \text { - each }\end{array} & 0.3125 & 0 \\ \begin{array}{l}\text { Power density }(\mathrm{mW} / \\ \left.\mathrm{cm}^{2}\right) \text { - each }\end{array} & 0.71 & 0\end{array}$

$\begin{array}{lll}\begin{array}{l}\text { Energy density }(\mathrm{J} / \\ \left.\mathrm{cm}^{2}\right)- \text { each }\end{array} & 0.162 & 0 \\ \text { Dose }(\mathrm{J}) \text { - each } & 0.07125 & 0 \\ \begin{array}{l}\text { Spot size of laser } \\ \left(\mathrm{cm}^{2}\right) \text { - each }\end{array} & 0.44 & 0.44\end{array}$

$\begin{array}{lll}\text { No of red LEDs } & 4 \text { Red } & 4 \text { Red } \\ \begin{array}{l}\text { Wavelength of red } \\ \text { LEDs }(\mathrm{nm})\end{array} & 640( \pm 10) & 640( \pm 10) \\ \text { Frequency }(\mathrm{Hz}) & 2 & 2\end{array}$

\begin{tabular}{|c|c|c|}
\hline $\begin{array}{l}\text { Average optical output } \\
(\mathrm{mW}) \text { - each }\end{array}$ & 15 & 1 \\
\hline $\begin{array}{l}\text { Power density (mW/ } \\
\left.\mathrm{cm}^{2}\right) \text {-each }\end{array}$ & 16.66 & 1.111 \\
\hline
\end{tabular}

\begin{tabular}{|c|c|c|}
\hline $\begin{array}{l}\text { Energy density }(\mathrm{J} / \\
\left.\mathrm{cm}^{2}\right) \text { - each }\end{array}$ & 3.80 & 0.253 \\
\hline Dose $(J)$ - each & 3.42 & 0.228 \\
\hline $\begin{array}{l}\text { Spot size of red LED } \\
\left(\mathrm{cm}^{2}\right)-\text { each }\end{array}$ & 0.9 & 0.9 \\
\hline No of infrared LEDs & 4 Infrared & 4 Infrared \\
\hline $\begin{array}{l}\text { Wavelength of infrared } \\
\text { LEDs }(\mathrm{nm})\end{array}$ & $875( \pm 10)$ & $875( \pm 10)$ \\
\hline Frequency (Hz) & 16 & 0 \\
\hline $\begin{array}{l}\text { Average optical output } \\
(\mathrm{mW}) \text { - each }\end{array}$ & 17.5 & 0 \\
\hline $\begin{array}{l}\text { Power density (mW/ } \\
\left.\mathrm{cm}^{2}\right) \text { - each }\end{array}$ & 19.44 & 0 \\
\hline $\begin{array}{l}\text { Energy density }(\mathrm{J} / \\
\left.\mathrm{cm}^{2}\right) \text { - each }\end{array}$ & 4.43 & 0 \\
\hline Dose $(\mathrm{J})$-each & 3.99 & 0 \\
\hline $\begin{array}{l}\text { Spot size of LED } \\
\left(\mathrm{cm}^{2}\right)-\text { each }\end{array}$ & 0.9 & 0.9 \\
\hline Magnetic field (mT) & 35 & 0 \\
\hline $\begin{array}{l}\text { Irradiation time per } \\
\text { site (s) }\end{array}$ & 228 & 228 \\
\hline Total dose per site $(\mathrm{J})$ & 30 & 0.912 \\
\hline $\begin{array}{l}\text { Total dose applied in } \\
\text { muscular group }(\mathrm{J})\end{array}$ & 180 & 5.47 \\
\hline $\begin{array}{l}\text { Aperture of device } \\
\left(\mathrm{cm}^{2}\right)\end{array}$ & 20 & 20 \\
\hline
\end{tabular}

Continued
Table 1 Continued

\begin{tabular}{|c|c|c|}
\hline & PBMT/sMF & Placebo \\
\hline Application mode & $\begin{array}{l}\text { Cluster probe } \\
\text { held stationary in } \\
\text { skin contact with } \\
\text { a } 90^{\circ} \text { angle and } \\
\text { slight pressure }\end{array}$ & $\begin{array}{l}\text { Cluster probe } \\
\text { held stationary in } \\
\text { skin contact with } \\
\text { a } 90^{\circ} \text { angle and } \\
\text { slight pressure }\end{array}$ \\
\hline
\end{tabular}

LEDs, light-emitting diodes; PBMT, photobiomodulation therapy; sMF, static magnetic field.

test and, secondarily, the Wilcoxon signed-rank test. Data will be analysed in terms of both their absolute values and their relationship to the percentage change based on the values established in the baseline tests. The significance level will be set at $p<0.05$. Cohen's $d$ will be used to calculate the effect sizes. ${ }^{42}$ The interpretation will be based on the values established by Cohen: small effect (0.2); moderate effect $(0.5)$ and large effect $(0.8) \cdot{ }^{42}$

\section{DISCUSSION}

The effects of PBMT/sMF on the maintenance of gains from a training protocol during a period of discontinuation of physical activity, that is, a detraining period, have not yet been investigated. We believe that PBMT/sMF will have a positive effect on the maintenance of gains from strength training during the detraining period. Given that many individuals, whether they are athletes or not, often fail to maintain training routines, the ability to minimise this loss would be of significant value in terms of accelerating the return to their previous fitness levels.

It is very likely that the groups receiving PBMT/sMF during the training protocol will present higher strength gains as shown in previous studies, ${ }^{23} 24$ and this can make data interpretation difficult. However, in this research project, we will analyse the outcomes in both absolute values and percentage of change compared with baseline, which can provide a clearer understanding of the primary and secondary outcomes of this study.

As strengths of this study we can highlight that it has high methodological quality since it is a randomised, controlled and prospectively registered clinical trial. Another strength is that it has a triple-blind design, which means that outcome assessors, therapists and patients will be blinded to interventions over the course of the study.

Additionally, the sample size was calculated based on a pilot study to provide the appropriate statistical power to detect precise differences for the primary outcome of the study. Finally, the doses, sites of application and the most effective point for application of PBMT/sMF were previously optimised and have been published in international peer-reviewed journals. ${ }^{18} 24$

\section{Limitations}

Although this study will analyse the effects of PBMT/ sMF on 4 weeks of detraining after 12 weeks of strength 
training, it is important to highlight that some sports-related injuries usually require $<4$ weeks of physical activity discontinuation. ${ }^{43-45}$ Therefore, the outcomes of this research project may be beneficial for decreasing the loss of strength during rehabilitation of such injuries.

It is also important to stress that such aspect never was investigated previously. And therefore, we believe that the present project is a major step towards the widespread use of PBMT/sMF as an ergogenic agent for not only increasing performance but also decreasing losses incurred by detraining. These factors have a direct impact on clinical practice when performing strength training is not possible, not only for athletes but also for patients in physical rehabilitation programme.

\section{ETHICS AND DISSEMINATION}

The study was approved by the Research Ethics Committee of Nove de Julho University (protocol number 1781602), furthermore this protocol was prospectively registered at ClinicalTrials.org. All volunteers will sign an informed consent at the time of enrolment in this study, all confidential patient data will be protected. Patient identity will not be disclosed.

The results from this study will be disseminated through scientific publications in international peer-reviewed journals and presentations at national and international scientific meetings.

\section{Author affiliations}

${ }^{1}$ Laboratory of Phototherapy and Innovative Technologies in Health (LaPIT), Postgraduate Program in Rehabilitation Sciences, Nove de Julho University, Sao Paulo, Brazil

${ }^{2}$ Masters and Doctoral Programs in Physical Therapy, Universidade Cidade de Sao Paulo, Sao Paulo, Brazil

${ }^{3}$ Postgraduate Program in Rehabilitation Sciences, Universidade Federal de Ciencias da Saude de Porto Alegre, Porto Alegre, Brazil

Contributors PRVdP, HLC and ECPL-J contributed to the concept and design of the study and established the hypothesis and wrote the original proposal. PRVdP, HLC, SST, CdSMM, AAV, BMB, PdTCdC and ECPL-J contributed significantly in creating the manuscript. SST and ECPL-J performed critical revisions of the manuscript. SST and ECPL-J wrote the final version of the manuscript. All authors read and approved the final version of the manuscript.

Funding This study is supported by São Paulo Research Foundation-FAPESP through research grants number 2016/11878-5 and 2018/21982-0, and by Brazilian Council of Science and Technology Development-CNPq 310281/2017-2.

Competing interests ECPL-J receives research support from Multi Radiance Medical (Solon, OH, USA), a PBMT/sMF device manufacturer. The remaining authors declare that they have no conflict of interests.

Patient consent for publication Not required.

Provenance and peer review Not commissioned; externally peer reviewed.

Open access This is an open access article distributed in accordance with the Creative Commons Attribution Non Commercial (CC BY-NC 4.0) license, which permits others to distribute, remix, adapt, build upon this work non-commercially, and license their derivative works on different terms, provided the original work is properly cited, appropriate credit is given, any changes made indicated, and the use is non-commercial. See: http://creativecommons.org/licenses/by-nc/4.0/.

\section{ORCID iD}

Ernesto Cesar Pinto Leal-Junior http://orcid.org/0000-0001-6393-7616
REFERENCES

1 Warburton DER, Nicol CW, Bredin SS. Health benefits of physical activity: the evidence. Can Med Assoc J 2006;174:801-9.

2 Warburton DER, Katzmarzyk PT, Rhodes RE, et al. EvidenceInformed physical activity guidelines for Canadian adults. Can $J$ Public Health 2007;98 Suppl 2:S16-68.

3 Willis LH, Slentz CA, Bateman LA, et al. Effects of aerobic and/ or resistance training on body mass and fat mass in overweight or obese adults. J Appl Physiol 2012;113:1831-7.

4 Pereira A, Izquierdo M, Silva AJ, et al. Muscle performance and functional capacity retention in older women after high-speed power training cessation. Exp Gerontol 2012;47:620-4.

5 Mangine GT, Hoffman JR, Gonzalez AM, et al. The effect of training volume and intensity on improvements in muscular strength and size in resistance-trained men. Physiol Rep 2015;3:e12472.

6 Lovell DI, Cuneo R, Wallace J, et al. The hormonal response of older men to sub-maximum aerobic exercise: the effect of training and detraining. Steroids 2012;77:413-8.

7 Stebbings GK, Morse Cl, McMahon GE, et al. Resting arterial diameter and blood flow changes with resistance training and detraining in healthy young individuals. J Athl Train 2013;48:209-19.

8 Leal-Junior ECP, Lopes-Martins Rodrigo Álvaro Brandão, Bjordal JM. Clinical and scientific recommendations for the use of photobiomodulation therapy in exercise performance enhancement and post-exercise recovery: current evidence and future directions. Braz J Phys Ther 2019;23:71-5.

9 Lopes-Martins RAB, Marcos RL, Leonardo PS, et al. Effect of lowlevel laser (Ga-Al-As $655 \mathrm{~nm}$ ) on skeletal muscle fatigue induced by electrical stimulation in rats. J Appl Physiol 2006;101:283-8.

10 Leal Junior ECP, Lopes-Martins RAB, Dalan F, et al. Effect of 655-nm low-level laser therapy on exercise-induced skeletal muscle fatigue in humans. Photomed Laser Surg 2008;26:419-24.

11 Leal Junior ECP, Lopes-Martins RAB, Vanin AA, et al. Effect of 830 $\mathrm{nm}$ low-level laser therapy in exercise-induced skeletal muscle fatigue in humans. Lasers Med Sci 2009;24:425-31.

12 Hemmings TJ, Kendall KL, Dobson JL. Identifying dosage effect of light-emitting diode therapy on muscular fatigue in quadriceps. $J$ Strength Cond Res 2017;31:395-402.

13 De Marchi T, Leal Junior ECP, Bortoli C, et al. Low-Level laser therapy (LLLT) in human progressive-intensity running: effects on exercise performance, skeletal muscle status, and oxidative stress. Lasers Med Sci 2012;27:231-6.

14 Rossato M, Dellagrana RA, Lanferdini FJ, et al. Effect of pre-exercise phototherapy applied with different cluster probe sizes on elbow flexor muscle fatigue. Lasers Med Sci 2016;31:1237-44.

15 Miranda EF, Vanin AA, Tomazoni SS, et al. Using pre-exercise photobiomodulation therapy combining super-pulsed lasers and light-emitting diodes to improve performance in progressive cardiopulmonary exercise tests. J Athl Train 2016;51:129-35.

16 Baroni BM, Leal Junior ECP, De Marchi T, et al. Low level laser therapy before eccentric exercise reduces muscle damage markers in humans. Eur J Appl Physiol 2010;110:789-96.

17 Baroni BM, Leal Junior ECP, Geremia JM, et al. Effect of lightemitting diodes therapy (LEDT) on knee extensor muscle fatigue. Photomed Laser Surg 2010;28:653-8.

18 Antonialli FC, De Marchi T, Tomazoni SS, et al. Phototherapy in skeletal muscle performance and recovery after exercise: effect of combination of super-pulsed laser and light-emitting diodes. Lasers Med Sci 2014;29:1967-76.

19 Aver Vanin A, De Marchi T, Tomazoni SS, et al. Pre-exercise infrared low-level laser therapy $(810 \mathrm{~nm})$ in skeletal muscle performance and postexercise recovery in humans, what Is the optimal dose? A randomized, double-blind, placebo-controlled clinical trial. Photomed Laser Surg 2016;34:473-82.

20 Pinto HD, Vanin AA, Miranda EF, et al. Photobiomodulation therapy improves performance and accelerates recovery of high-level rugby players in field test: a randomized, crossover, double-blind, placebocontrolled clinical study. J Strength Cond Res 2016;30:3329-38.

21 Dornelles MP, Fritsch CG, Sonda FC, et al. Photobiomodulation therapy as a tool to prevent hamstring strain injuries by reducing soccer-induced fatigue on hamstring muscles. Lasers Med Sci 2019;34:1177-84.

22 De Marchi T, Leal-Junior ECP, Lando KC, et al. Photobiomodulation therapy before futsal matches improves the staying time of athletes in the Court and accelerates post-exercise recovery. Lasers Med Sci 2019;34:139-48.

23 Baroni BM, Rodrigues R, Freire BB, et al. Effect of low-level laser therapy on muscle adaptation to knee extensor eccentric training. Eur J Appl Physiol 2015;115:639-47.

24 Vanin AA, Miranda EF, Machado CSM, et al. What is the best moment to apply phototherapy when associated to a strength 
training program? A randomized, double-blinded, placebo-controlled trial : Phototherapy in association to strength training. Lasers Med Sci 2016;31:1555-64.

25 Miranda EF, Tomazoni SS, de Paiva PRV, et al. When is the best moment to apply photobiomodulation therapy (PBMT) when associated to a treadmill endurance-training program? A randomized, triple-blinded, placebo-controlled clinical trial. Lasers Med Sci 2018;33:719-27.

26 Leal-Junior ECP, Vanin AA, Miranda EF, et al. Effect of phototherapy (low-level laser therapy and light-emitting diode therapy) on exercise performance and markers of exercise recovery: a systematic review with meta-analysis. Lasers Med Sci 2015;30:925-39.

27 Vanin AA, Verhagen E, Barboza SD, et al. Photobiomodulation therapy for the improvement of muscular performance and reduction of muscular fatigue associated with exercise in healthy people: a systematic review and meta-analysis. Lasers Med Sci 2018;33:181-214.

28 Machado AF, Micheletti JK, Lopes JSS, et al. Phototherapy on management of creatine kinase activity in general versus localized exercise: a systematic review and meta-analysis. Clin J Sport Med 2018. doi:10.1097/JSM.0000000000000606. [Epub ahead of print: 21 Jun 2018].

29 Nakano J, Kataoka H, Sakamoto J, et al. Low-Level laser irradiation promotes the recovery of atrophied gastrocnemius skeletal muscle in rats. Exp Physiol 2009;94:1005-15.

30 Brooks NE, Myburgh KH. Skeletal muscle wasting with disuse atrophy is multi-dimensional: the response and interaction of myonuclei, satellite cells and signaling pathways. Front Physiol 2014;5:99.

31 Kadi F, Schjerling P, Andersen LL, et al. The effects of heavy resistance training and detraining on satellite cells in human skeletal muscles. J Physiol 2004;558:1005-12.

32 Joensen J, Demmink JH, Johnson Ml, et al. The thermal effects of therapeutic lasers with 810 and $904 \mathrm{~nm}$ wavelengths on human skin. Photomed Laser Surg 2011;29:145-53.
33 Grandinétti VdosS, Miranda EF, Johnson DS, et al. The thermal impact of phototherapy with concurrent super-pulsed lasers and red and infrared LEDs on human skin. Lasers Med Sci 2015;30:1575-81.

34 Brown L. Isokinetics in human performance. Champaign. IL: Human Kinetics, 2000: 16-31.

35 Irving BA, Rutkowski J, Brock DW, et al. Comparison of Borg- and $\mathrm{OMNI}-\mathrm{RPE}$ as markers of the blood lactate response to exercise. Med Sci Sports Exerc 2006;38:1348-52.

36 Abe Tet al. Whole body muscle hypertrophy from resistance training: distribution and total mass. Br J Sports Med 2003;37:543-5.

37 Blazevich AJ, Gill ND, Zhou S. Intra- and intermuscular variation in human quadriceps femoris architecture assessed in vivo. J Anat 2006;209:289-310.

38 Kubo K, Kanehisa H, Azuma K, et al. Muscle architectural characteristics in women aged 20-79 years. Med Sci Sports Exerc 2003;35:39-44.

39 Miyatani $\mathrm{M}$, Kanehisa $\mathrm{H}$, Ito $\mathrm{M}$, et al. The accuracy of volume estimates using ultrasound muscle thickness measurements in different muscle groups. Eur J Appl Physiol 2004;91:264-72.

40 Baroni BM, Rodrigues R, Franke RA, et al. Time course of neuromuscular adaptations to knee extensor eccentric training. Int $J$ Sports Med 2013;34:904-11.

41 Baroni BM, Geremia JM, Rodrigues R, et al. Muscle architecture adaptations to knee extensor eccentric training: rectus femoris vs. vastus lateralis. Muscle Nerve 2013;48:498-506.

42 Cohen J. Statistical power analysis for the behavioral sciences. 2nd ed. Hillsdale (NJ): Routledge, 1988: 400.

43 Herzog MM, Mack CD, Dreyer NA, et al. Ankle Sprains in the National Basketball association, 2013-2014 through 2016-2017. Am J Sports Med 2019;47:2651-8.

44 Cuñado-González Álvaro, Martín-Pintado-Zugasti A, RodríguezFernández Ángel L, et al. Prevalence and factors associated with injuries in elite Spanish volleyball. J Sport Rehabil 2019;14:2651-8.

45 Ekstrand J, Krutsch W, Spreco A, et al. Time before return to play for the most common injuries in professional football: a 16-year followup of the UEFA elite Club injury study. Br J Sports Med 2019:bjsp orts-2019-100666. 